\title{
Quality of life in people living with HIV: a cross-sectional study in Ouagadougou, Burkina Faso
}

Fidèle Bakiono ${ }^{1 *}$, Laurent Ouédraogo ${ }^{2,3}$, Mahamoudou Sanou², Sékou Samadoulougou', Patrice Wendpouiré Laurent Guiguemdé ${ }^{2}$ Fati Kirakoya-Samadoulougou ${ }^{1}$ and Annie Robert ${ }^{1}$

\begin{abstract}
HIV/AIDS is a leading cause of death in most of sub-Saharan countries. HIV/AIDS impact on the quality of life of persons living with HIV in Burkina Faso hasn't been well documented. The aim of the study was to assess the quality of life in persons living with HIV and its associated factors.

A cross-sectional study was conducted in Ouagadougou. 424 persons living with HIV were included in the study according to their status with regard to Highly Active Anti Retroviral Treatment: 115 were not yet under treatment, 21 started the treatment within the three months preceding the enrolment and 288 were under treatment for at least 12 months. The quality of life was assessed through the WHOQOL HIV-BREF. Statistical comparisons were made using Mann Whitney $\mathrm{U}$ test, Kruskal-Wallis $\mathrm{H}$ test, Pearson's khi2 or Fisher's exact test. Correlations were appreciated using Spearman's rho. Logistic regression was used to examine associations between the quality of life scores and sociodemographic or clinical variables.

The mean global score of quality of life in all patients was 82.4. Better scores were recorded in the spiritual domain and worst scores in the environmental domain. Men had a higher global score than women $(p<0.001)$. Illiteracy was significantly associated with a lower quality of life $(p=0.001)$. Patients having support for medical treatment had a significantly better quality of life $(p<0.01)$. In multivariate analysis, being a man, having a support for medical care, getting older and self-perceived as healthy, were associated with a global score of quality of life higher than 77 , that corresponds to the mid-range of the score in our data.

These findings suggest the importance of the socio-psychological support and of a good environment in order to improve the quality of life of people living with HIV, especially in women, in younger and in those having no support for medical care. In the environmental domain, actions of HIV services providers should focus on better accessibility to social and health care, promotion of income-generating activities especially for women and youth living with HIV.
\end{abstract}

Keywords: Quality of life; HIV/AIDS; Burkina Faso; WHOQOL HIV-BREF

\section{Background}

In developing countries, especially in Western subSaharan Africa, leading causes of years of life lost are malaria, lower respiratory infections, diarrheal diseases and HIV/AIDS (Lopez et al. 2006). Since the introduction of Highly Active Antiretroviral Treatment (HAART) in the

\footnotetext{
* Correspondence: f.bakiono@yahoo.fr

'Pôle Epidémiologie et Biostatistique, Institut de Recherche Expérimentale et Clinique (IREC), Faculté de Santé Publique, Université catholique de Louvain, Clos Chapelle-aux-Champs 30, 1200 Brussels, Belgium

Full list of author information is available at the end of the article
}

mid 1990's, treatments reduce the morbidity, the mortality related to HIV and HIV transmission (Hayward 2013). Longevity has been improved, but side effects of treatments are reported (Gakhar et al. 2013; Duran et al. 2001). The quality of life becomes an important area of concern for patients, health care providers, psychological and social support providers (Solomon et al. 2009). Studies conducted in sub Saharan Africa showed an improvement of the quality of life over time in people initiating treatment (Stangl et al. 2007; Jelsma et al. 2005). In Burkina Faso, where the prevalence in the general population is $1.1 \%$

\section{实


(UNAIDS 2012), HIV remains a public health problem with large variations across subgroups(INSD/ICF International 2012; Kirakoya-Samadoulougou et al. 2013; Kirakoya-Samadoulougou et al. 2014). Efforts are made nationwide to improve the health and the wellbeing of people living with HIV (CNLS-IST/BF 2011). But there is few scientific evidence about their health related quality of life. One study conducted in Burkina Faso showed an evident increase in quality of life in people initiating the treatment over a 12 months follow-up time (Jaquet et al. 2013). But the quality of life of other people waiting for treatment or under treatment for more than 12 months remains insufficiently documented.

The aim of our study was to assess the quality of life in persons living with HIV and its associated factors.

\section{Methods}

A cross-sectional study was conducted in Ouagadougou in September 2012. Recruitment took place in five structures that take care of People Living With HIV/AIDS (PLWHA) in the city. In their records, 22,788 persons were followed up for medical care and sociopsychological support. They represent $73.44 \%$ of the people followed up by all facilities in the Central Region, of which Ouagadougou is the capital city. Two of these facilities are integrated in the national health system and three are communitybased. Those integrated in the national health system are the District Hospital of Boulmiougou (DHB) and the "Hôpital du Jour" a service of University Hospital Yalgado Ouédraogo (UHYO). The three communitybased organizations are the Center for Information, Counseling and Documentation on AIDS and Tuberculosis (CICDoc), the Association African Solidarity (AAS), the Association Laafi la Viim (ALAVI). All those facilities offer anonymous voluntary counseling and testing (VCT) services, prevention counseling, medical treatment and monitoring, nutrition counseling, psychological and social support.

\section{Sampling}

Setting the level of confidence at $95 \%$, with a $5 \%$ precision with a maximal uncertainty, the required sample size was 385 . This number was increased by $10 \%$ (39 people) leading to a total of 424 persons recruited. Determining the number of subjects to be recruited in each facility was made according to the size of the active list in each structure. Active list is defined as the number of PLWHA under treatment or not, and followed by the structure. Thus, in the district hospital of Boulmiougou which represents $35.38 \%$ of the total number of people followed by the 5 structures, 150 people were recruited. At the Hôpital du Jour (UHYO), representing 31.84\% of this number, 135 persons were recruited. In the CICDoc, AAS and ALAVI representing respectively $15.10 \%, 9.20 \%$ and $8.49 \%$ of the active list of the five structures, 64, 39 and 36 persons were recruited, respectively. In each structure, to insure a sound representation of people living with HIV, recruitment took place from the routine consultation, and people were classified into three groups according to the status linked to the HAART: 1) HIV positive but not under HAART yet; 2) people who started HAART within the three months preceding the enrolment and 3) people under HAART for at least 1 year. The number in each group was determined in proportion to its size in the active list. Those who met criteria and who agreed to participate in the study were interviewed. We used trained interviewers, due to the high illiterate rate in the country and that is $43.7 \%$ in urban area of Burkina Faso (INSD 2008).

\section{Quality of life assessment tool}

To assess the quality of life, we used the French version of WHOQOL HIV-BREF (Preau et al. 2007; Marcellin et al. 2007). For people who didn't understand French, Mooré version of WHOQOL HIV-BREF developed by Bakiono et al. was used. Mooré is the main language of communication in the country, whatever residence of individuals we consider (rural or urban) (INSD 2008). WHOQOL HIV-BREF is a 31-item tool which asks interviewee to rate his quality of life in many ways, during the last two weeks preceding the interview. WHOQOL HIV-BREF explores six domains of the quality of life : physical, psychological, level of independence, social relationships, environment and spirituality. Answers are rated on a five-point Likert scale from 1 to 5 . Scoring was performed according to recommendations of the World Health Organization Quality of Life group (WHO 2002). Higher scores indicate better quality of life. In our sample, the Cronbach's alpha for reliability assessment was 0.85 , indicating good internal consistency of the tool.

\section{Characteristics collected}

Beside the data collected to assess the quality of life, socio demographic, economic and clinical data were collected by trained interviewers, fluent in French and in Mooré. Characteristics collected were: gender, age, marital status, occupation, level of education, knowledge attitudes and practices, risk behaviors, duration under treatment, time since being HIV-positive tested, serological status, great part in monthly expenses, disclosure of HIV status, experience in stigmatization.

\section{Statistical analysis}

Analyses were performed using IBM SPSS 19.0. Groups were compared using Mann Whitney U test or KruskalWallis $\mathrm{H}$ test for continuous variables and Pearson's $X^{2}$ or Fisher's exact p-values for discrete or qualitative variables. Bivariate correlations were appreciated using the $\rho$ of Spearman. For all analyses, a p-value lower than 0.05 was 
considered as significant. A cut off of global score of quality of life was set at 77, which represents the mid-range of the scale based on our data. A global score less than or equal to 77 was considered as bad and a global score higher than 77 as good. The robustness of the cut-off point was tested by comparing results using other cut-offs like 1) observed percentile 67 (upper tertile) which is data driven; 2) upper third of the scale which is not data driven and corresponds to $24+2(120-24) / 3=88$. Variables associated to good global score of quality of life in a bivariate analysis $(\mathrm{p}<0.15)$ were included into a multiple logistic regression model.

\section{Ethical concerns}

The present study has been approved by the Ethics Committee for Health Research from the Ministry of Health of Burkina Faso. All patients who agreed to participate in the study signed freely an informed consent.

\section{Results}

Patients were $37.6 \pm 8.5$ years of age on average. $96.7 \%$ of them lived in Ouagadougou for an average time of $23 \pm$ 14 years. The mean duration of HIV infection reported by interviewees was $5.7 \pm 4.5$ years and the mean time since initiation of HAART was $5.0 \pm 3.1$ years. Knowledge, attitudes and practices of interviewees have been assessed and showed that $5 \%$ of them didn't know transmission ways of HIV and 4.2\% didn't know how to prevent from HIV. $69.5 \%$ of them didn't know the difference between AIDS and HIV positive. Among literate interviewees, the proportion of persons who didn't know the difference between AIDS and HIV positive was $60.4 \%$ while among illiterate interviewees, it was $88.3 \%(\mathrm{p}<0.001)$. Despite their status, $14.4 \%$ of the interviewees never used condom when having sex, 25.1\% used it sometimes and $12.4 \%$ of them had no sex any longer. Among interviewees, $78.1 \%$ were asymptomatic, $18.5 \%$ were symptomatic and $3.4 \%$ were at AIDS stage. $35.6 \%$ didn't know how they contracted HIV while 57.3\% reported heterosexual intercourse. $72.9 \%$ of respondents were taking Highly Active Antiretroviral Treatment (HAART). Characteristics of interviewees are shown in Table 1.

The mean (SD) global score of quality of life in all patients was 82.4 (10.8). According to the domain, mean scores were 14.8(2.7) for physical domain; 13.6(2.7) for psychological domain; 13.3(2.5) for the level of independence domain; $13.4(2.9)$ for social relationships domain; 10.8(2.2) for environmental domain and 16.3(2.6) for religious and personal believes domain. In patients not under HAART, the global score of quality of life was 80.7(9.6). In patients under treatment for less than 3 months it was 80.2(14.2) and it was 80.2(10.9) for patients under treatment for at least 1 year $(p=0.07)$. Men had a global score of 87.2( 9.7) while women had a global score of $81.7(10.8)$ $[p<0.001]$. Sharing or not one's status with a family member was not significantly associated with better quality of life. Hospital or community-based nature of the structure of care had no significant impact on the quality of life of people living with HIV. Illiteracy was significantly associated with a lower quality of life $(p=0.001)$. Having support for medical treatment of the subject was significantly associated with better quality of life $(p<0.01)$. The perception of being ill was associated with poorer quality of life. Different domains scores and the overall score of quality of life related to socio-demographic and clinical characteristics are shown in Tables 2 and 3.

In our sample, overall general health perception was significantly and positively correlated with all domains of the quality of life, as for global score of quality of life $(p<0.01)$.

In multivariate analysis, being a man, having a support for medical care, older age and self-perceived as healthy, were associated with a global score of quality of life higher than 77 (Table 4). Being a woman, self perceived as ill result with lower score of quality of life (Table 5).

When using a slightly cut-off point of 88 , that corresponds to the upper third of the scale, associations were comparable (Gender effect: $\mathrm{OR}=0.37$ changed to $\mathrm{OR}=0.43$, self perceived as ill effect: $\mathrm{OR}=0.31$ changed to $\mathrm{OR}=0.43$ ). Associations also maintained when reducing the cut-off point.

\section{Discussion}

Our study involved 424 persons living with HIV. A majority of them were small traders, employees in informal sector and housewives. According to national data in urban area, like Ouagadougou, the population is mainly working in services and in trading (INSD 2008), which is consistent with our findings. The most common route of transmission reported by interviewees was heterosexual intercourse which is consistent with data from subSaharan Africa and specifically general data of Burkina Faso, reporting heterosexual intercourse as the main route of transmission (UNAIDS 2012).

A majority of our patients declared to be asymptomatic: $76.6 \%$. It was almost the same proportion which was under treatment. Free access to treatment could have enhanced the proportion of persons under treatment leading to a better health status, without symptoms.

Our study showed that $14.4 \%$ of respondents never used condoms when having sex and $25.1 \%$ of them used it sometimes. These proportion of sexual risk behavior contrasts with the proportion of respondents who knew the routes of transmission of HIV, which was $95 \%$. These sexual risk behaviors were highlighted by Guira et al. in their study on sexuality and the risk of sexual transmission (Guira et al. 2013). In their study involving 87 heterosexual serodiscordant couples, it was found that while $97.5 \%$ of couples were aware of the use of condoms as a mean of preventing the HIV transmission, nearly $60 \%$ of them did not use a condom systematically. The privacy concerns 
Table 1 Characteristics of $\mathbf{4 2 4}$ persons living with HIV in Ouagadougou, Burkina Faso

\begin{tabular}{lcc}
\hline Interviewees characteristics & $\begin{array}{c}\text { Proportion } \\
(\%)\end{array}$ & $\begin{array}{c}\text { Mean } \pm \text { SD } \\
\text { Median [IQR] }\end{array}$ \\
\hline Age (years) & & $37.6 \pm 8.5$ \\
Gender & & \\
$\quad$ Men & 12.5 & \\
$\quad$ Women & 87.5 &
\end{tabular}

Marital status

Single

Separated/divorced

Widow

Concubine

Married

Matrimonial system

Monogamous

Polygamous

Profession

Public or private sector employee $\quad 16.0$

Trader or informal sector $\quad 50.7$

Housewife 23.6

Cultivator

Student or pupil

Jobless

Religion

$\begin{array}{ll}\text { Muslim } & 43.0 \\ \text { Catholic } & 42.6\end{array}$

Protestant

Level of education

Illiterate

32.8

Primary school

31.8

Secondary school

University

Time since HIV-positive tested (years)

HIV transmission route reported

Heterosexual intercourse

Don't know

35.6

Other

7.2

Highly active antiretroviral treatment status

$$
\text { No treatment }
$$

Under treatment for less than 3 months

Under treatment for at least 1 year HAART duration (years)
Table 1 Characteristics of 424 persons living with HIV in Ouagadougou, Burkina Faso (Continued)

\begin{tabular}{lc}
\hline Serology status & \\
Asymptomatic & 78.1 \\
Symptomatic & 18.5 \\
AIDS & 3.4 \\
HIV services providers & \\
DHB & 35.4 \\
HUYO & 31.8 \\
CIC_DOC & 15.1 \\
AAS & 9.2 \\
ALAVI & 8.5
\end{tabular}

Household size

$5[3 ; 7]$

Status disclosure with relative

$\begin{array}{ll}\text { No } & 18.6 \\ \text { Yes } & 81.4\end{array}$

Sexual behavior risk

No risk $\quad 60.5$

Risk $\quad 39.5$

Accepted by the family

No $\quad 11.1$

Yes $\quad 88.9$

Greater part of monthly expenses

$\begin{array}{lc}\text { Food } & 73.0 \\ \text { Health } & 10.3 \\ \text { Housing } & 7.9 \\ \text { Other } & 8.8\end{array}$

$\mathrm{DHB}=$ District Hospital of Boulmiougou; $\mathrm{HUYO}=$ University Hospital Yalgado Ouédraogo; $\mathrm{CICDoc}=$ Center for Information, Counseling and Documentation on AIDS and Tuberculosis; AAS = Association African Solidarity; ALAVI = Association Laafi la Viim.

and the desire to give birth (for women) were the main reasons for the inconsistent use of condoms.

$5.7 \pm 4.4$ In our sample, the global score of quality of life was 82.4 \pm 10.8 . It is a lower mean score than what has been found in Ethiopia by Deribew et al. with a mean score set at 91.9 in HIV-positive people (Deribew et al. 2009; Deribew et al. 2013). In these studies mean score was 80.3 in co-infected HIV-TB people, close to our score. In Vietnam, Tran reported 76.2, a slightly lower mean score of overall quality of life than our mean value, but close to the cut-off value we used in the present study (Tran et al. 2012).

Whatever domain of quality of life we consider, scores were higher among men compared to women. These higher scores recorded in men may be explained by the socioeconomic level of men, generally higher than that of women, facilitating a better dealing with the disease. However, our results regarding quality of life and gender suggest more investigations. In rural Uganda, Stangl et al. 
Table 2 Quality of life domains scores by demographic characteristics of persons living with HIV in Ouagadougou, Burkina Faso $(n=424)$

\begin{tabular}{|c|c|c|c|c|c|c|c|}
\hline \multirow{2}{*}{$\begin{array}{l}\text { Socio-demographic } \\
\text { characteristics }\end{array}$} & \multicolumn{6}{|c|}{ Domain of quality of life } & \multirow{2}{*}{$\begin{array}{c}\text { Global } \\
\text { score of } \\
\text { Quality Of } \\
\text { Life (Mean } \pm \text { SD) }\end{array}$} \\
\hline & $\begin{array}{c}\text { Physical } \\
\text { domain } \\
(\text { Mean } \pm \text { SD) }\end{array}$ & $\begin{array}{l}\text { Psychological } \\
\text { domain } \\
(\text { Mean } \pm \text { SD) }\end{array}$ & $\begin{array}{c}\text { Level of } \\
\text { independence } \\
(\text { Mean } \pm \text { SD) }\end{array}$ & $\begin{array}{c}\text { Social } \\
\text { relationships } \\
\text { (Mean } \pm \text { SD) }\end{array}$ & $\begin{array}{c}\text { Environmental } \\
\text { domain } \\
(\text { Mean } \pm \text { SD) }\end{array}$ & $\begin{array}{c}\begin{array}{c}\text { Spiritual } \\
\text { domain } \\
(\text { Mean } \pm \text { SD) }\end{array}\end{array}$ & \\
\hline Gender & $p=0.14$ & $p=0.01$ & $p=0.008$ & $p=0.003$ & $p=0.001$ & $p=0.03$ & $p<0.001$ \\
\hline Men $(n=53)$ & $15.4 \pm 2.3$ & $14.4 \pm 2.7$ & $14.1 \pm 2.7$ & $14.5 \pm 2.5$ & $11.7 \pm 2.1$ & $16.9 \pm 2.5$ & $87.2 \pm 9.7$ \\
\hline Women $(n=370)$ & $14.7 \pm 2.7$ & $13.5 \pm 2.6$ & $13.2 \pm 2.4$ & $13.2 \pm 2.9$ & $10.7 \pm 2.1$ & $16.2 \pm 2.6$ & $81.7 \pm 10.8$ \\
\hline HIV services provider & $p=0.22$ & $p=0.34$ & $p=0.003$ & $p=0.01$ & $p<0.001$ & $p=0.53$ & $p=0.05$ \\
\hline AAS $(n=39)$ & $15.8 \pm 2.4$ & $14.4 \pm 2.7$ & $13.7 \pm 2.2$ & $13.3 \pm 2.7$ & $11.0 \pm 1.8$ & $16.3 \pm 2.7$ & $84.7 \pm 10.3$ \\
\hline $\mathrm{DHB}(\mathrm{n}=150)$ & $14.9 \pm 2.7$ & $13.3 \pm 2.6$ & $13.7 \pm 2.6$ & $14.0 \pm 2.7$ & $11.4 \pm 2.2$ & $16.2 \pm 2.7$ & $83.7 \pm 10.9$ \\
\hline CIC-Doc $(n=64)$ & $14.6 \pm 2.7$ & $13.5 \pm 2.5$ & $13.5 \pm 2.6$ & $13.3 \pm 3.0$ & $11.1 \pm 1.9$ & $15.9 \pm 2.4$ & $82.2 \pm 11.1$ \\
\hline ALAVI $(n=36)$ & $14.7 \pm 2.9$ & $13.6 \pm 2.7$ & $13.0 \pm 2.7$ & $13.4 \pm 3.1$ & $9.9 \pm 2.3$ & $16.5 \pm 3.0$ & $81.2 \pm 11.3$ \\
\hline HUYO $(n=135)$ & $14.7 \pm 2.7$ & $13.6 \pm 2.8$ & $12.7 \pm 2.2$ & $12.7 \pm 2.9$ & $10.2 \pm 2.1$ & $16.4 \pm 2.4$ & $80.6 \pm 10.3$ \\
\hline Level of education & $p=0.02$ & $p=0.17$ & $p<0.001$ & $p=0.07$ & $p=0.31$ & $p=0.49$ & $p=0.007$ \\
\hline Illiterate $(n=139)$ & $14.3 \pm 2.6$ & $13.3 \pm 2.7$ & $12.5 \pm 2.3$ & $12.9 \pm 2.7$ & $10.5 \pm 2.0$ & $16.2 \pm 2.6$ & $79.9 \pm 10.1$ \\
\hline Primary school $(n=135)$ & $15.0 \pm 2.6$ & $13.7 \pm 2.6$ & $13.6 \pm 2.1$ & $13.7 \pm 2.7$ & $11.0 \pm 2.2$ & $16.4 \pm 2.6$ & $83.6 \pm 10.4$ \\
\hline Secondary school $(n=135)$ & $15.1 \pm 2.8$ & $13.9 \pm 2.7$ & $13.7 \pm 2.8$ & $13.5 \pm 3.1$ & $10.9 \pm 2.3$ & $16.3 \pm 2.5$ & $83.6 \pm 11.6$ \\
\hline University $(n=15)$ & $15.6 \pm 1.8$ & $12.9 \pm 2.6$ & $13.9 \pm 2.2$ & $13.7 \pm 3.1$ & $11.0 \pm 2.4$ & $15.1 \pm 3.2$ & $82.3 \pm 10.3$ \\
\hline Marital status & $p=0.49$ & $p=0.13$ & $p=0.55$ & $p=0.01$ & $p=0.73$ & $p=0.87$ & $p=0.53$ \\
\hline Single $(n=62)$ & $14.4 \pm 3.1$ & $13.1 \pm 3.1$ & $13.4 \pm 2.8$ & $13.2 \pm 3.4$ & $10.6 \pm 2.7$ & $16.0 \pm 3.0$ & $80.9 \pm 13.2$ \\
\hline Married $(n=150)$ & $15.0 \pm 2.5$ & $13.9 \pm 2.3$ & $13.5 \pm 2.1$ & $13.8 \pm 2.7$ & $10.9 \pm 2.1$ & $16.3 \pm 2.3$ & $83.7 \pm 9.8$ \\
\hline Partner $(n=40)$ & $15.0 \pm 2.4$ & $13.1 \pm 3.4$ & $13.2 \pm 2.6$ & $13.6 \pm 3.0$ & $10.5 \pm 2.2$ & $16.1 \pm 2.5$ & $81.6 \pm 11.6$ \\
\hline Widower $(n=89)$ & $14.5 \pm 2.8$ & $14.0 \pm 2.3$ & $13.1 \pm 2.3$ & $13.0 \pm 2.4$ & $10.8 \pm 2.0$ & $16.4 \pm 2.7$ & $82.0 \pm 9.7$ \\
\hline Divorced/Separated $(n=83)$ & $15.1 \pm 2.7$ & $13.1 \pm 2.7$ & $13.1 \pm 2.9$ & $12.9 \pm 3.0$ & $10.9 \pm 2.0$ & $16.3 \pm 2.5$ & $81.7 \pm 11.1$ \\
\hline Matrimonial system & $p=0.17$ & $p=0.15$ & $p=0.16$ & $p=0.27$ & $p=0.07$ & $p=0.83$ & $p=0.16$ \\
\hline Monogamous ( $n=99$ ) & $15.1 \pm 2.3$ & $14.0 \pm 2.4$ & $13.2 \pm 2.2$ & $13.9 \pm 2.4$ & $11.0 \pm 2.0$ & $16.4 \pm 2.2$ & $83.9 \pm 9.2$ \\
\hline Polygamous ( $n=37$ ) & $14.4 \pm 2.8$ & $13.5 \pm 2.2$ & $13.6 \pm 1.6$ & $13.4 \pm 2.9$ & $10.4 \pm 2.1$ & $16.1 \pm 2.8$ & $81.6 \pm 10.1$ \\
\hline Religion & $p=0.96$ & $p=0.39$ & $p=0.35$ & $p=0.21$ & $p=0.08$ & $p=0.006$ & $p=0.78$ \\
\hline Muslims ( $n=182)$ & $14.9 \pm 2.7$ & $13.6 \pm 2.6$ & $13.3 \pm 2.3$ & $13.6 \pm 2.8$ & $10.9 \pm 2.2$ & $16.1 \pm 2.4$ & $82.7 \pm 10.5$ \\
\hline Catholics $(n=180)$ & $14.8 \pm 2.6$ & $13.4 \pm 2.6$ & $13.2 \pm 2.7$ & $13.2 \pm 2.7$ & $10.9 \pm 2.0$ & $16.1 \pm 2.7$ & $81.9 \pm 10.6$ \\
\hline Protestants $(n=61)$ & $14.8 \pm 2.8$ & $13.9 \pm 3.0$ & $13.7 \pm 2.2$ & $13.1 \pm 3.5$ & $10.3 \pm 2.4$ & $17.1 \pm 2.6$ & $83.2 \pm 10.7$ \\
\hline Profession & $p=0.10$ & $p<0.005$ & $p=0.001$ & $p=0.03$ & $p=0.30$ & $p=0.16$ & $p=0.007$ \\
\hline Public, private employees $(n=68$ ) & $15.6 \pm 2.3$ & $14.3 \pm 2.2$ & $14.5 \pm 2.2$ & $13.7 \pm 3.0$ & $11.3 \pm 2.2$ & $16.6 \pm 2.6$ & $86.3 \pm 9.5$ \\
\hline Trade \& Informal sector $(n=215)$ & $14.8 \pm 2.7$ & $13.6 \pm 2.5$ & $13.2 \pm 2.4$ & $13.2 \pm 2.8$ & $10.7 \pm 2.1$ & $16.2 \pm 2.6$ & $82.0 \pm 10.2$ \\
\hline
\end{tabular}


Table 2 Quality of life domains scores by demographic characteristics of persons living with HIV in Ouagadougou, Burkina Faso $(\mathrm{n}=424)($ Continued)

\begin{tabular}{|c|c|c|c|c|c|c|c|}
\hline Housewives $(n=100)$ & $14.6 \pm 2.5$ & $13.3 \pm 2.6$ & $13.1 \pm 2.2$ & $13.7 \pm 2.7$ & $10.8 \pm 2.2$ & $16.4 \pm 2.4$ & $82.0 \pm 10.5$ \\
\hline Farmers $(n=13)$ & $14.3 \pm 3.3$ & $14.9 \pm 2.2$ & $12.7 \pm 2.3$ & $14.4 \pm 2.1$ & $10.9 \pm 2.6$ & $17.0 \pm 2.4$ & $84.6 \pm 11.7$ \\
\hline Student \& pupil $(n=12)$ & $13.9 \pm 3.4$ & $10.9 \pm 3.7$ & $12.3 \pm 3.6$ & $13.1 \pm 3.6$ & $10.1 \pm 2.5$ & $14.3 \pm 3.4$ & $74.8 \pm 16.5$ \\
\hline Jobless $(n=16)$ & $14.0 \pm 3.7$ & $12.1 \pm 4.1$ & $12.4 \pm 4.1$ & $11.3 \pm 3.3$ & $10.9 \pm 1.7$ & $15.8 \pm 2.6$ & $76.7 \pm 14.2$ \\
\hline Family cohesion & $p=0.03$ & $p=0.02$ & $p=0.40$ & $p=0.02$ & $p=0.005$ & $p=0.001$ & $p=0.001$ \\
\hline Hassle $(n=75)$ & $14.3 \pm 2.9$ & $12.9 \pm 2.7$ & $13.1 \pm 2.6$ & $12.7 \pm 3.2$ & $10.2 \pm 2.4$ & $15.4 \pm 2.7$ & $78.7 \pm 11.2$ \\
\hline Without hassle $(n=343)$ & $15.0 \pm 2.6$ & $13.7 \pm 2.6$ & $13.3 \pm 2.5$ & $13.5 \pm 2.8$ & $11.0 \pm 2.1$ & $16.5 \pm 2.5$ & $83.3 \pm 10.5$ \\
\hline
\end{tabular}
ALAVI = Association Laafi la Viim. 
Table 3 Quality of life domains scores by clinical characteristics of persons living with HIV in Ouagadougou, Burkina Faso ( $n=424 *$ )

\begin{tabular}{|c|c|c|c|c|c|c|c|}
\hline \multirow[t]{2}{*}{ Clinical characteristics } & \multicolumn{6}{|c|}{ Domain of quality of life } & \multirow{2}{*}{$\begin{array}{c}\text { Global score } \\
\text { of quality } \\
\text { of life } \\
\text { (Mean } \pm \text { SD) }\end{array}$} \\
\hline & $\begin{array}{l}\text { Physical domain } \\
(\text { Mean } \pm \text { SD) }\end{array}$ & $\begin{array}{l}\text { Psychological } \\
\text { domain } \\
(\text { Mean } \pm \text { SD) }\end{array}$ & $\begin{array}{c}\text { Level of } \\
\text { independence } \\
(\text { Mean } \pm \text { SD) }\end{array}$ & $\begin{array}{l}\text { Social relationships } \\
\quad(\text { Mean } \pm \text { SD) }\end{array}$ & $\begin{array}{l}\text { Environmental } \\
\text { domain } \\
(\text { Mean } \pm \text { SD) }\end{array}$ & $\begin{array}{l}\text { Spiritual domain } \\
(\text { Mean } \pm S D)\end{array}$ & \\
\hline Serology status & $p=0.003$ & $p=0.52$ & $p=0.001$ & $p=0.32$ & $p=0.40$ & $p=0.15$ & $p=0.02$ \\
\hline Asymptomatic $(n=325)$ & $15.1 \pm 2.5$ & $13.7 \pm 2.5$ & $13.5 \pm 2.4$ & $13.5 \pm 2.8$ & $10.9 \pm 2.2$ & $16.4 \pm 2.5$ & $83.4 \pm 10.3$ \\
\hline Symptomatic $(n=77)$ & $14.0 \pm 2.7$ & $13.3 \pm 3.1$ & $12.4 \pm 2.7$ & $13.0 \pm 2.8$ & $10.6 \pm 2.2$ & $16.2 \pm 2.4$ & $79.7 \pm 11.5$ \\
\hline AIDS $(n=14)$ & $14.2 \pm 3.1$ & $13.0 \pm 2.8$ & $14.2 \pm 2.8$ & $13.5 \pm 3.4$ & $10.7 \pm 2.1$ & $14.7 \pm 3.4$ & $80.5 \pm 12.1$ \\
\hline Highly Active Antiretroviral Treatment status & $p=0.002$ & $P<0.001$ & $p=0.92$ & $p=0.44$ & $p=0.87$ & $p=0.78$ & $p=0.08$ \\
\hline No treatment $(n=110)$ & $14.2 \pm 2.7$ & $12.8 \pm 2.6$ & $13.2 \pm 2.4$ & $13.6 \pm 2.4$ & $10.9 \pm 2.1$ & $16.3 \pm 2.3$ & $82.8 \pm 11.1$ \\
\hline Under treatment $(n=312)$ & $15.1 \pm 2.6$ & $13.9 \pm 2.6$ & $13.3 \pm 2.5$ & $13.3 \pm 3.0$ & $10.8 \pm 2.2$ & $16.2 \pm 2.7$ & $81.1 \pm 9.7$ \\
\hline Perceived health state & $p<0.001$ & $p<0.001$ & $p<0.001$ & $p<0.001$ & $p=0.001$ & $p=0.005$ & $p<0.001$ \\
\hline Good health state $(n=270)$ & $15.5 \pm 2.4$ & $14.1 \pm 2.4$ & $13.8 \pm 2.2$ & $13.8 \pm 2.8$ & $11.1 \pm 2.2$ & $16.6 \pm 2.4$ & $85.2 \pm 9.7$ \\
\hline Median health state $(n=131)$ & $13.8 \pm 2.7$ & $12.9 \pm 2.7$ & $12.8 \pm 2.5$ & $12.8 \pm 2.7$ & $10.4 \pm 1.9$ & $15.8 \pm 2.8$ & $78.8 \pm 10.5$ \\
\hline Bad health state $(n=23)$ & $12.0 \pm 1.8$ & $10.9 \pm 2.8$ & $10.2 \pm 3.0$ & $11.5 \pm 3.2$ & $9.8 \pm 1.9$ & $15.2 \pm 2.7$ & $69.9 \pm 10.6$ \\
\hline Perceived disease & $p<0.001$ & $p<0.001$ & $p<0.001$ & $p=0.007$ & $p=0.01$ & $p=0.01$ & $p<0.001$ \\
\hline Yes $(n=142)$ & $13.7 \pm 2.8$ & $12.6 \pm 2.9$ & $12.3 \pm 2.6$ & $12.8 \pm 2.9$ & $10.5 \pm 2.2$ & $15.8 \pm 2.7$ & $77.9 \pm 11.3$ \\
\hline No $(n=244)$ & $15.5 \pm 2.4$ & $14.3 \pm 2.4$ & $13.8 \pm 2.2$ & $13.7 \pm 2.8$ & $11.0 \pm 2.1$ & $16.5 \pm 2.5$ & $85.1 \pm 9.6$ \\
\hline Sexual behavior & $p=0.04$ & $p=0.32$ & $p=0.82$ & $p=0.93$ & $p=0.12$ & $p=0.52$ & $p=0.35$ \\
\hline Risk $(n=165)$ & $14.5 \pm 2.6$ & $13.5 \pm 2.5$ & $13.3 \pm 2.3$ & $13.3 \pm 3.0$ & $10.6 \pm 2.3$ & $16.4 \pm 2.3$ & $81.9 \pm 10.6$ \\
\hline No risk $(n=253)$ & $15.0 \pm 2.7$ & $13.6 \pm 2.8$ & $13.3 \pm 2.6$ & $13.4 \pm 2.8$ & $11.0 \pm 2.1$ & $16.1 \pm 2.8$ & $82.7 \pm 11.0$ \\
\hline Support for medical care & $p=0.16$ & $p=0.28$ & $p=0.55$ & $p<0.001$ & $p=0.57$ & $p=0.04$ & $p=0.02$ \\
\hline Yes $(n=266)$ & $15.0 \pm 2.7$ & $13.7 \pm 2.6$ & $13.4 \pm 2.3$ & $13.8 \pm 2.7$ & $10.9 \pm 2.1$ & $16.5 \pm 2.5$ & $83.4 \pm 10.4$ \\
\hline No $(n=157)$ & $14.6 \pm 2.7$ & $13.4 \pm 2.8$ & $13.1 \pm 2.7$ & $12.6 \pm 3.1$ & $10.7 \pm 2.2$ & $15.9 \pm 2.7$ & $80.5 \pm 11.2$ \\
\hline
\end{tabular}

*Total may differ according to characteristics. 
Table 4 Factors associated with a higher value of global score of the quality of life in persons living with HIV in Ouagadougou, Burkina Faso

\begin{tabular}{|c|c|c|c|c|c|}
\hline & Total $\mathrm{n}^{*}$ & $\begin{array}{l}\text { Global score of } \\
\text { QOL >77 n (\%) }\end{array}$ & $\begin{array}{c}\text { Univariate Unadjusted } \\
\text { OR }(95 \% \mathrm{Cl})\end{array}$ & $\begin{array}{c}\text { Multivariate Adjusted } \\
\text { OR }(95 \% \mathrm{Cl})\end{array}$ & $\begin{array}{c}P \text { for } \\
\text { Adjusted OR }\end{array}$ \\
\hline \multicolumn{6}{|l|}{ Conjugal life } \\
\hline Alone & 234 & $160(68.4)$ & 1 & & \\
\hline In couple & 190 & $146(76.8)$ & $1.53(0.99-2.37)$ & & \\
\hline \multicolumn{6}{|l|}{ Gender } \\
\hline Man & 53 & $45(84.9)$ & 1 & 1 & \\
\hline Woman & 370 & $260(70.3)$ & $0.42(0.19-0.92)$ & $0.37(0.15-0.88)$ & 0.02 \\
\hline \multicolumn{6}{|l|}{ Support for medical care } \\
\hline No & 157 & $104(66.2)$ & 1 & 1 & \\
\hline Yes & 266 & $201(75.6)$ & $1.57(1.02-2.43)$ & $2.18(1.32-3.61)$ & 0.002 \\
\hline \multicolumn{6}{|l|}{ Age (year) } \\
\hline $19-24$ & 26 & $12(46.2)$ & 1 & 1 & 0.01 \\
\hline $25-34$ & 135 & $90(66.7)$ & $2.33(0.99-5.5)$ & $2.68(1.04-6.89)$ & 0.04 \\
\hline $35-44$ & 170 & $129(75.9)$ & $3.67(1.57-8.56)$ & $4.04(1.55-10.51)$ & 0.004 \\
\hline $45-65$ & 91 & $73(80.2)$ & $4.73(1.87-11.96)$ & $4.31(1.52-12.22)$ & 0.006 \\
\hline \multicolumn{6}{|l|}{ Nature of care facility } \\
\hline Hospital & 285 & $200(70.2)$ & 1 & & \\
\hline Community based & 139 & $106(76.3)$ & $1.35(0.85-2.17)$ & & \\
\hline \multicolumn{6}{|c|}{ Health expense as greater part of monthly spending } \\
\hline No & 72 & $45(62.5)$ & 1 & & \\
\hline Yes & 352 & $261(74.1)$ & $1.72(1.00-2.93)$ & & \\
\hline \multicolumn{6}{|c|}{ Highly Active Antiretroviral Treatment } \\
\hline No & 110 & $72(65.5)$ & 1 & & \\
\hline Yes & 312 & $233(74.7)$ & $1.55(0.97-2.48)$ & & \\
\hline \multicolumn{6}{|l|}{ School } \\
\hline No & 139 & $93(66.9)$ & 1 & & \\
\hline Yes & 285 & $213(74.7)$ & 1.46 & & \\
\hline \multicolumn{6}{|l|}{ Self perceived as ill } \\
\hline No & 244 & $197(80.7)$ & 1 & 1 & \\
\hline Yes & 142 & 79 (55.6) & $0.29(0.18-0.47)$ & $0.31(0.19-0.50)$ & $<0.001$ \\
\hline
\end{tabular}

*Total number may differ according to factor.

didn't find any significant difference in the quality of life according to gender (Stangl et al. 2012). In Nigeria, no difference has been found in quality of life scores in regard with gender (Ogbuji and Oke 2010). In Burkina Faso, Jaquet et al. found a slight difference with advantage for men in mental health summary score and advantage for women in physical health summary score (Jaquet et al. 2013). In a large literature review on quality of life across countries and continents, Chandra et al. showed that in most of them, women reported lower quality of life than men (Chandra et al. 2009). These results, which included patients at different stages of the disease and under treatment, are consistent with our findings.
In our findings, the highest scores were recorded in the spiritual domain and the lowest scores were found in the environmental domain. In Burkina Faso, 99.6\% of the population practice a religion (INSD 2008). As religions recommend the seek of heaven's wellbeing, instead of earthy happiness, religion practices of our subjects and the distress situation in which they are living due to the disease could explain the high scores recorded in the spiritual domain. This demonstrates how people in distress refer to religion to better accept their situation. In a study conducted in Ethiopia (Deribew et al. 2013), better scores were recorded in spiritual domain and the worst in environmental scores, results consistent with ours. The lower scores in environmental domain could 
Table 5 Multivariate logistic regression model for predicting chances of a global score of quality of life higher than $\mathbf{7 7}$ in persons living with HIV in Ouagadougou, Burkina Faso

\begin{tabular}{|c|c|c|}
\hline Variables & $\beta \pm S E$ & P-value \\
\hline Living in couple (yes $=1 ;$ no $=0$ ) & $0.32 \pm 0.27$ & 0.23 \\
\hline Being a woman (yes $=1 ;$ no $=0$ ) & $-0.80 \pm 0.46$ & 0.08 \\
\hline Having support for medical care (yes $=1 ;$ no $=0$ ) & $0.65 \pm 0.27$ & 0.01 \\
\hline \multicolumn{3}{|l|}{ Age (years) } \\
\hline $25-34($ yes $=1 ;$ no $=0)$ & $0.90 \pm 0.49$ & 0.06 \\
\hline $35-44($ yes $=1 ;$ no $=0)$ & $1.30 \pm 0.50$ & 0.01 \\
\hline $45-65($ yes $=1 ;$ no $=0)$ & $1.42 \pm 0.55$ & 0.01 \\
\hline Hospital nature of care facility (yes $=1 ;$ no $=0$ ) & $0.05 \pm 0.27$ & 0.85 \\
\hline Health expense as greater part of monthly spending (yes $=1$; no $=0$ ) & $0.25 \pm 0.30$ & 0.41 \\
\hline Under highly active antiretroviral treatment (yes $=1 ; n o=0$ ) & $0.16 \pm 0.28$ & 0.56 \\
\hline Having been to school (yes $=1 ; \mathrm{no}=0$ ) & $0.26 \pm 0.26$ & 0.32 \\
\hline Self perceived as ill (yes $=1 ;$ no $=0$ ) & $-1.12 \pm 0.25$ & $<0.001$ \\
\hline Constant & $-0.02 \pm 0.77$ & 0.97 \\
\hline
\end{tabular}

be explained by the economical situation of our respondents dominated by small traders, employees of informal sector and housewives. As environmental domain explores, among others, home environment, financial resources, health and social care accessibility and quality, the lower economic level in our sample can explain lower scores in that domain.

Our study showed that in the HIV/AIDS care clinics, people living with HIV were predominantly under treatment. But they were still $27 \%$ not yet under treatment. Starting treatment for people living with HIV is based on the level of CD4 and viral load (CNLS-IST/BF 2008). However, in Burkina Faso, starting treatment may be delayed for financial reasons. In January 2010 the country started with free treatment for people in need; a rapid growth in the number of people on treatment has subsequently been observed. Prior to 2010, a significant decrease in the price of Highly Active Antiretroviral Treatments has been conceded in 2005 followed by another decrease in 2008 (Kouanda et al. 2010). In our study, patients who started treatment before 2005 represented $20 \%$ of the number of people under treatment. The large majority was under treatment since 2005. These results suggest that the high cost of treatment may be the reason of the small proportion of people on treatment by 2005 , explaining at the same time the average duration of the initiation of treatment towards 5 years.

Our results didn't show significantly higher scores according to the duration under treatment except for spirituality domain where being under treatment since 2005 showed better scores than before 2005. Handajani et al. in their study involving 88 outpatients living with HIV showed a significant association between duration of HAART and physical domain (Handajani et al. 2012). In Uganda, Bajunirwe et al. showed that duration of less than one year was significantly associated with a lower physical health summary (Bajunirwe et al. 2009).

Our findings highlighted that being a man, having a support for medical care, ageing and self-perceived as healthy were associated with a global score of quality of life higher than 77. In a previous study in Burkina Faso, authors found that older age ( $>=35$ years) et gender (be a man) were associated with better mental health summary score (Jaquet et al. 2013). Our results are also consistent with the study of Skevington et al. with regard to age (Skevington 2012). No matter the domain of quality of life, scores they found were better in older people. Adewuya et al. also found in Nigeria that lower education level and poor social support were associated with poor quality of life (Adewuya et al. 2008).

\section{Limitations}

The whole country has about 100 structures for taking care of people living with HIV. Our study was conducted in Ouagadougou, the capital city of Burkina Faso, in five HIV services providers and during a one-month enrolment period. Results may not reflect nationwide overview, especially in rural areas. However, these five structures were chosen for their large queues of people followed up and they are a good representation of people living with HIV in Ouagadougou or in urban areas of Burkina Faso.

\section{Conclusion}

To our knowledge, it is the first time quality of life in persons living with HIV has been assessed using WHOQOL HIV, without restricting to people initiating anti retroviral 
treatment in Burkina Faso. Our findings showed worst scores in the environmental domain, with better scores of quality of life associated with good family cohesion, with a support for medical care, with a self-perceived as healthy, with ageing and in man. These findings highlight the importance of the socio-psychological support and of a good environment in order to improve the quality of life of people living with HIV, especially in women, at a younger age and in those having no support for medical care. In the environmental domain, actions of HIV services providers should focus on a better accessibility to social and health care, on the promotion of income-generating activities, especially for women and youth living with HIV.

\begin{abstract}
Abbreviations
AAS: Association African Solidarity; ALAVI: Association Laafi la Viim; CICDoc: Center for Information, Counseling and Documentation on AIDS and Tuberculosis; DHB: District Hospital of Boulmiougou; HAART: Highly Active Antiretroviral Treatment; HIV/AIDS: Human Immunodeficiency Virus/Acquired Immunodeficiency Syndrome; PLWHA: People Living With HIV/AIDS; UHYO: University Hospital Yalgado Ouédraogo; VCT: Voluntary Counseling and Testing; WHO: World Health Organization; WHOQOL: World Health Organization Quality of Life; WHOQOL HIV-BREF: World Health Organization Quality of Life assessment brief tool in patients with Human Immunodeficiency Virus infection.
\end{abstract}

\section{Competing interests}

The authors declare they have no competing interest.

\section{Authors' contributions}

$F B$, LO and AR contributed to conception of the study. FB, MS and PWLG carried out the data collection. FB drafted the manuscript. FB, SS and FS-K contributed to data analysis. All authors discussed the results, read and approved the final manuscript.

\section{Acknowledgements}

We thank all investigators, interviewees and the staff of the following institutions for their contribution to this study: District Hospital of Boulmiougou; Department of internal Medicine, University Hospital Yalgado Ouedraogo; Center for Information, Counseling and Documentation on AIDS and Tuberculosis, Ouagadougou; the Association African Solidarity, Ouagadougou; the Association Laafi la Viim, Ouagadougou.

\section{Author details}

'Pôle Epidémiologie et Biostatistique, Institut de Recherche Expérimentale et Clinique (IREC), Faculté de Santé Publique, Université catholique de Louvain, Clos Chapelle-aux-Champs 30, 1200 Brussels, Belgium. ${ }^{2}$ Unité de Formation et de Recherche en Sciences de la santé, Université de Ouagadougou, 03 BP 7021 Ouagadougou 03, Burkina Faso. ${ }^{3}$ Institut Régional de Santé Publique de Ouidah, BP 384 Ouidah, Bénin.

Received: 14 May 2014 Accepted: 15 July 2014

Published: 21 July 2014

\section{References}

Adewuya AO, Afolabi MO, Ola BA, Ogundele OA, Ajibare AO, Oladipo BF, Fakande I (2008) Relationship between depression and quality of life in persons with HIV infection in Nigeria. Int J Psychiatry Med 38(1):43-51

Bajunirwe F, Tisch DJ, King CH, Arts EJ, Debanne SM, Sethi AK (2009) Quality of life and social support among patients receiving antiretroviral therapy in Western Uganda. AIDS Care 21(3):271-279

Chandra PS, Satyanarayana VA, Satishchandra P, Satish KS, Kumar M (2009) Do men and women with HIV differ in their quality of life? A study from South India. AIDS Behav 13(1):110-117

CNLS-IST/BF (2008) Guidelines for medical care of people living with HIV in Burkina Faso. CMLS, Ouagadougou
CNLS-IST/BF (2011) Cadre stratégique de lutte contre le VIH/Sida et les IST 2011-2015. Ouagadougou-Burkina Faso

Deribew A, Deribe K, Reda AA, Tesfaye M, Hailmichael Y, Maja T, Colebunders R (2013) Change in quality of life: a follow up study among patients with HIV infection with and without TB in Ethiopia. BMC Public Health 13:408, doi:10.1186/1471-2458-13-408

Deribew A, Tesfaye M, Hailmichael Y, Negussu N, Daba S, Wogi A, Belachew T, Apers $\mathrm{L}$, Colebunders R (2009) Tuberculosis and HIV co-infection: its impact on quality of life. Health Qual Life Outcomes 7:105, doi:10.1186/1477-7525-7-105

Duran S, Saves M, Spire B, Cailleton V, Sobel A, Carrieri P, Salmon D, Moatti JP, Leport C (2001) Failure to maintain long-term adherence to highly active antiretroviral therapy: the role of lipodystrophy. AIDS 15(18):2441-2444

Gakhar H, Kamali A, Holodniy M (2013) Health-related quality of life assessment after antiretroviral therapy: a review of the literature. Drugs 73(7):651-672

Guira O, Tieno H, Sawadogo S, Drabo JY (2013) Sexuality and risk for sexual transmission of HIV among serodiscordant couples in Ouagadougou, Burkina Faso. Bull Soc Pathol Exot 106(1):43-47

Handajani YS, Djoerban Z, Irawan H (2012) Quality of life people living with HIV/AIDS: outpatient in Kramat 128 Hospital Jakarta. Acta Medica Indonesiana 44(4):310-316

Hayward P (2013) Highlights from the seventh IAS Conference. Lancet Infect Dis 13(8):659

INSD (2008) Recensement général de la population et de l'habitat (RGPH) de 2006 du Burkina Faso - Résultats définitifs. INSD, Ouagadougou

INSD/ICF International (2012) Enquête démographique et de santé et à indicateurs multiples du Burkina Faso. INSD \& ICF International, Calverton Mayland, USA

Jaquet A, Garanet F, Balestre E, Ekouevi DK, Azani JC, Bognounou R, Dah E, Kondombo JC, Dabis F, Drabo J, collaboration aftIWA (2013) Antiretroviral treatment and quality of life in Africans living with HIV: 12-month follow-up in Burkina Faso. J Int AIDS Soc 16:18867

Jelsma J, Maclean E, Hughes J, Tinise X, Darder M (2005) An investigation into the health-related quality of life of individuals living with HIV who are receiving HAART. AIDS Care 17(5):579-588

Kirakoya-Samadoulougou F, Fao P, Yaro S, Defer MC, Meda N, Robert A, Nagot N (2014) Are healthcare workers at higher risk of HIV infection than the general population in Burkina Faso? Epidemiol Infect 142(3):662-670

Kirakoya-Samadoulougou F, Nagot N, Yaro S, Fao P, Defer MC, Ilboudo F, Langani Y, Meda N, Robert A (2013) Are teachers at higher risk of HIV infection than the general population in Burkina Faso? Int J STD AIDS 24(8):651-659

Kouanda S, Bocoum FY, Doulougou B, Bila B, Yameogo M, Sanou MJ, Sawadogo M, Sondo B, Msellati P, Desclaux A (2010) User fees and access to ARV treatment for persons living with HIV/AIDS: implementation and challenges in Burkina Faso, a limited-resource country. AIDS Care 22(9):1146-1152

Lopez AD, Mathers CD, Ezzati M, Jamison DT, Murray CJ (2006) Global and regional burden of disease and risk factors, 2001: systematic analysis of population health data. Lancet 367(9524):1747-1757

Marcellin F, Preau M, Ravaux I, Dellamonica P, Spire B, Carrieri MP (2007) Self-reported fatigue and depressive symptoms as main indicators of the quality of life (QOL) of patients living with HIV and Hepatitis C: implications for clinical management and future research. HIV Clin Trials 8(5):320-327

Ogbuji QC, Oke AE (2010) Quality of life among persons living with HIV infection in Ibadan, Nigeria. Afr J Med Med Sci 39(2):127-135

Preau M, Apostolidis T, Francois C, Raffi F, Spire B (2007) Time perspective and quality of life among HIV-infected patients in the context of HAART. AIDS Care 19(4):449-458

Skevington SM (2012) Is quality of life poorer for older adults with HIV/AIDS? International evidence using the WHOQOL-HIV. AIDS Care 24(10):1219-1225

Solomon S, Batavia A, Venkatesh KK, Brown L, Verma P, Cecelia AJ, Daly C, Mahendra VS, Kumarasamy N, Mayer KH (2009) A longitudinal quality-of-life study of HIV-infected persons in South India: the case for comprehensive clinical care and support services. AIDS Educ Prev 21(2):104-112

Stangl AL, Bunnell R, Wamai N, Masaba H, Mermin J (2012) Measuring quality of life in rural Uganda: reliability and validity of summary scores from the medical outcomes study HIV health survey (MOS-HIV). Qual Life Res 21(9):1655-1663

Stangl AL, Wamai N, Mermin J, Awor AC, Bunnell RE (2007) Trends and predictors of quality of life among HIV-infected adults taking highly active antiretroviral therapy in rural Uganda. AIDS Care 19(5):626-636 
Tran BX, Ohinmaa A, Nguyen LT, Oosterhoff P, Vu PX, Vu TV, Larsson M (2012) Gender differences in quality of life outcomes of HIV/AIDS treatment in the latent feminization of HIV epidemics in Vietnam. AIDS Care 24(10):1187-1196 UNAIDS (2012) Global report: UNAIDS report on the global AIDS epidemic., Available at http://www.unaids.org/en/media/unaids/contentassets/documents/epidemiology/ 2012/gr2012/20121120_UNAIDS_Global_Report_2012_with_annexes_en.pdf. Accessed 12 July 2013

WHO (2002) WHOQOL-HIV instrument users manual. Scoring and coding for the WHOQOL-HIV instruments. WHO Press, Geneva, Switzerland

doi:10.1186/2193-1801-3-372

Cite this article as: Bakiono et al:: Quality of life in people living with

HIV: a cross-sectional study in Ouagadougou, Burkina Faso. SpringerPlus 2014 3:372

\section{Submit your manuscript to a SpringerOpen ${ }^{\circ}$} journal and benefit from:

- Convenient online submission

- Rigorous peer review

- Immediate publication on acceptance

- Open access: articles freely available online

- High visibility within the field

- Retaining the copyright to your article

Submit your next manuscript at $\gg$ springeropen.com 